\title{
Effects of dietary supplementation with $\alpha$-linolenic acid on the phospholipid fatty acid composition and quality of spermatozoa in cockerel from 24 to 72 weeks of age
}

\author{
K. A. Kelso ${ }^{1}$, S. Cerolini ${ }^{2}$, B. K. Speake ${ }^{1 *}$, L. G. Cavalchini ${ }^{3}$ and \\ R. C. Noble \\ ${ }^{1}$ Department of Biochemical Sciences, Scottish Agricultural College, Auchincruive, Ayr, KA6 5HW, UK; \\ ${ }^{2}$ Istituto Difesa Valorizzazione Germoplasma Animale, Consiglio Nazionale delle Ricerche, via Celoria 10, \\ 20133 Milano, Italy; and 'Istituto di Zootecnica, Universita' di Milano, via Celoria 10, 20133 Milano, \\ Italy
}

\begin{abstract}
The aim of this study was to investigate the effects of supplementing the diet of the male chicken with $\alpha$-linolenic acid (18:3n-3) on the phospholipid fatty acid composition, motility and fertilizing ability of chicken spermatozoa. The birds in the control group received a diet supplemented with soybean oil rich in linoleic acid (18:2n-6) whereas those in the test group were supplemented with linseed oil rich in $\alpha$-linolenic acid. A number of age-related changes in the lipid parameters of the spermatozoa were observed in control birds. Between 24 and 72 weeks of age the amount of total lipid in the spermatozoa of control birds increased by approximately 2.4 times and the proportions of cholesterol and free fatty acid also increased significantly, whereas the proportions of phospholipid and triacylglycerol decreased. In addition, the proportion of phosphatidylcholine in the total phospholipid increased, whereas the proportion of phosphatidylserine decreased during the same period. The proportion of docosatetraenoic acid $(22: 4 n-6)$ in the phospholipid decreased significantly between 24 and 72 weeks of age. The concentration of spermatozoa in the semen of control birds increased to a maximum at week 39 and had decreased significantly by week 72 . Supplementation with $\alpha$-linolenic acid had little or no effect on the proportion of docosahexaenoic acid (22:6n-3) in the phospholipid profile of the spermatozoa. However, supplementation with $\alpha$-linolenic acid did produce a significant but small increase in the proportion of docosapentaenoic acid (22:5n-3) recorded at 39 and 54 weeks. Thus, this study shows that the fatty acid composition of the sperm phospholipid demonstrates a marked resistance to dietary manipulation. Supplementation with $\alpha$-linolenic acid significantly enhanced semen fertility at week 39 . The results suggest that the small increase in the proportion of n-3 fatty acids in the sperm phospholipids induced by enriching the diet with $\alpha$-linolenic acid is associated with a significant improvement in semen quality at 39 weeks of age.
\end{abstract}

\section{Introduction}

The phospholipids of mammalian spermatozoa display a highly characteristic fatty acid composition, the most striking feature of which is the presence of extremely high proportions of docosahexaenoic acid (22:6n-3) (Neill and Masters, 1972; Poulos and White, 1973; Poulos et al., 1973; Scott, 1973; Jain and Anand, 1976; Salem et al., 1986; Lin et al., 1993). This long-chain, highly polyunsaturated fatty acid is also present at very high concentrations in the phospholipids of the retina and brain (Salem ef al., 1986; Neuringer et al., 1988). In contrast to mammals, spermatozoa from avian species that have so far

${ }^{*}$ Correspondence.

Received 14 August 1996. been studied display extremely low concentrations of docosahexaenoic acid in their phospholipids. Instead, the major fatty acyl components of avian spermatozoa are arachidonic $(20: 4 \mathrm{n}-6)$ and docosatetraenoic (22:4n-6) acids (Darin-Bennett et al., 1974; Howarth et al., 1977; Ravie and Lake, 1985; Kelso et al., 1996). Thus, avian spermatozoa are characterized by high amounts of $\mathrm{C}_{20-22}$ polyunsaturates of the $\mathrm{n}-6$ series, whereas long-chain fatty acids of the $\mathrm{n}-3$ series predominate in mammalian spermatozoa. However, it should be noted that the compositional data for avian spermatozoa are almost wholly confined to domestic poultry species (the chicken and the turkey) and that little information is available for birds living in the wild. Since commercial poultry production currently relies on compounded feeds that generally contain a vast excess of n-6 fatty acids (mainly linoleic acid (18:2n-6)) over $n-3$ fatty 
acids (Noble, 1986), it is conceivable that the reported fatty acid composition of avian spermatozoa is a dietary-induced displacement from the 'natural' pattern rather than a true phylogenetic difference from mammals.

It is well known that animals deprived of a source of $n-3$ fatty acids at critical stages of embryonic or neonatal development display greatly reduced concentrations of $22: 6 n-3$ in brain and retina phospholipids and, by way of compensation, accumulate high proportions of long-chain $\mathrm{n}-6$ fatty acids such as 22:4n-6 and 22:5n-6 (Salem et al., 1986; Neuringer et al., 1988; Anderson et al., 1989). Thus, the characteristic fatty acid profiles of poultry spermatozoa are typical of those associated with $n-3$ deficiency. There is considerable evidence that 22:6n-3 performs an essential role in the functional maturation of tissues such as the brain and retina and that its replacement by $22: 4 n-6$ and $22: 5 n-6$ during n-3 deficiency is unable to prevent a range of functional impairments (Neuringer et al., 1988; Sanders et al., 1988; Innis, 1991). Similarly, it appears that the 22:6n-3 present in mammalian spermatozoa also performs an essential function in promoting optimal fertility, as marked reductions in the amount of this fatty acid in spermatozoa are associated with impaired sperm number, motility and fertilizing ability (Nissan et al., 1981; Nissan and Kreysel, 1983). If the typical fatty acid composition currently observed for the spermatozoa of domestic poultry is simply an aberration due to a dietary imbalance of $n-3$ versus n-6 fatty acids, the fertility of poultry might be amenable to improvement via appropriate dietary manipulation.

\section{Materials and Methods}

\section{Dietary supplementation of cockerels and semen collection}

Male chickens (Cobb broiler-breeder strain) were purchased at 21 days of age from a commercial poultry supplier. The birds were housed in single cages in a controlled environment with a photoperiod of $13 \mathrm{~h}$ light: $11 \mathrm{~h}$ dark. They were each fed $130 \mathrm{~g}$ feed day ${ }^{-1}$ containing $12.5 \%(\mathrm{w} / \mathrm{w})$ crude protein and $11.5 \mathrm{MJ}$ metabolizable energy $\mathrm{kg}^{-1}$. From 23 to 72 weeks of age, 15 birds received the control diet, which was supplemented with soybean oil rich in linoleic acid (18:2n-6) $(6 \%$ $(w / w)$ of feed); another 15 birds received the diet supplemented with linseed oil rich in $\alpha$-linolenic acid $(18: 3 n-3)(6 \%(w / w)$ of feed). The total fat content of both diets was $9 \%(w / w)$ of feed. The fatty acid compositions of the two diets are shown (Table 1). Both diets contained vitamin $\mathrm{E}$ at a concentration of $40 \mathrm{mg} \mathrm{kg}^{-1}$ feed. Fresh batches of the respective feeds were produced weekly and regular analysis revealed that no significant changes in the fatty acid compositions occurred throughout the duration of the experiment. There were no significant effects of dietary supplementation with 18:3n-3 on the growth and body masses of the birds, for example, at 72 weeks of age the mean body masses ( $\mathrm{g}$ ) of the control and supplemented birds were $4555 \pm 429 \mathrm{~g}$ and $4654 \pm 384 \mathrm{~g}(n=15)$, respectively.

The birds were trained for semen collection (Lake and Stewart, 1978) from 21 weeks of age. Two semen samples were collected per week after this age, apart from the weeks chosen for analysis $(24,39,54$ and 72 weeks of age) when three samples were taken.
Table 1. Fatty acid compositions of the control diet supplemented with soybean oil rich in linoleic acid (18:2n-6) and of the diet supplemented with linseed oil rich in $\alpha$-linolenic acid $(18: 3 n-3)$ in male chickens

\begin{tabular}{lrr}
\hline & \multicolumn{2}{c}{ Supplementation } \\
\cline { 2 - 3 } Fatty acids $(\%, w / w)$ & Soybean oil & Linseed oil \\
\hline $16: 0$ & $12.1 \pm 0.3$ & $8.7 \pm 0.2$ \\
$18: 0$ & $3.5 \pm 0.1$ & $3.3 \pm 0.1$ \\
$18: 1 n-9$ & $23.4 \pm 0.1$ & $20.7 \pm 0.2$ \\
$18: 2 n-6$ & $52.9 \pm 1.9$ & $28.8 \pm 0.3$ \\
$18: 3 n-3$ & $6.2 \pm 0.1$ & $36.8 \pm 1.5$ \\
Ratio of $18: 2 n-6$ to $18: 3 n-3$ & $8.5 \pm 0.2$ & $0.78 \pm 0.1$ \\
\hline
\end{tabular}

Values are means $\pm \mathrm{SD}$ of measurements taken from four samples of feed. Only the major fatty acids $(>0.5 \%, w / w)$ are shown.

No $\mathrm{C}_{20-22}$ fatty acids were detected (limits of detection were approximately $0.1 \%, w / w)$.

\section{Evaluation of semen quality}

Within $20 \mathrm{~min}$ of collection, the semen volume was measured using a graduated tube, and sperm concentration and motility were determined using a Cellsoft Computer-Assisted Digital Imaging System (CellSoft (MM) Version 3, Cryoresources Ltd, New York). Motility was expressed as the percentage of the total number of spermatozoa that were motile. The fertilizing ability of the semen was assessed by artificially inseminating laying hens. The hens (ISA-Warren strain) were purchased at 21 weeks of age, housed in single cages in a controlled environment of $16 \mathrm{~h}$ light: $8 \mathrm{~h}$ dark and fed freely with a standard layer diet (11.5 MJ metabolizable energy $\mathrm{kg}^{-1}$ feed and $18 \%(\mathrm{w} / \mathrm{w})$ crude protein). At each time of insemination, the males and females were the same age. Semen samples were diluted with two volumes of $0.9 \%(\mathrm{w} / \mathrm{w}) \mathrm{NaCl}$ and a single dose of spermatozoa (approximately $7 \times 10^{7}$ ) was inseminated within $20 \mathrm{~min}$ of semen collection. Semen from groups of three males was pooled to provide five replicate pooled samples per dietary treatment. Each pooled sample of semen was used to inseminate seven hens, and eggs were collected over the next seven days. At each of the four ages studied, hens were selected at random to receive semen from either the control or the supplemented males. The eggs were incubated at $37.5^{\circ} \mathrm{C}$ and $60 \%$ relative humidity and were turned automatically for 7 days. The eggs were then opened and the numbers of those containing live, dead or no embryos were recorded. Only those containing no embryos were classed as infertile.

\section{Lipid extraction and analysis}

Semen samples from groups of three cockerels were pooled to obtain sufficient material for lipid analysis, five replicate pooled samples were used for each of the dietary treatments. Semen samples were diluted with an equal volume of $0.85 \%$ $(\mathrm{w} / \mathrm{v}) \mathrm{NaCl}$ and centrifuged at $700 \mathrm{~g}$ for $20 \mathrm{~min}$ at $4^{\circ} \mathrm{C}$. The upper diluted seminal plasma layer was discarded; the wash procedure was repeated with $\mathrm{I} \mathrm{ml} 0.85 \%(\mathrm{w} / \mathrm{v}) \mathrm{NaCl}$ solution 
Table 2. Characteristics of semen from cockerels on a control diet rich in linoleic acid (18:2n-6) and from birds on a diet supplemented with $\alpha$-linolenic acid (18:3n-3) at $24,39,54$ and 72 weeks of age

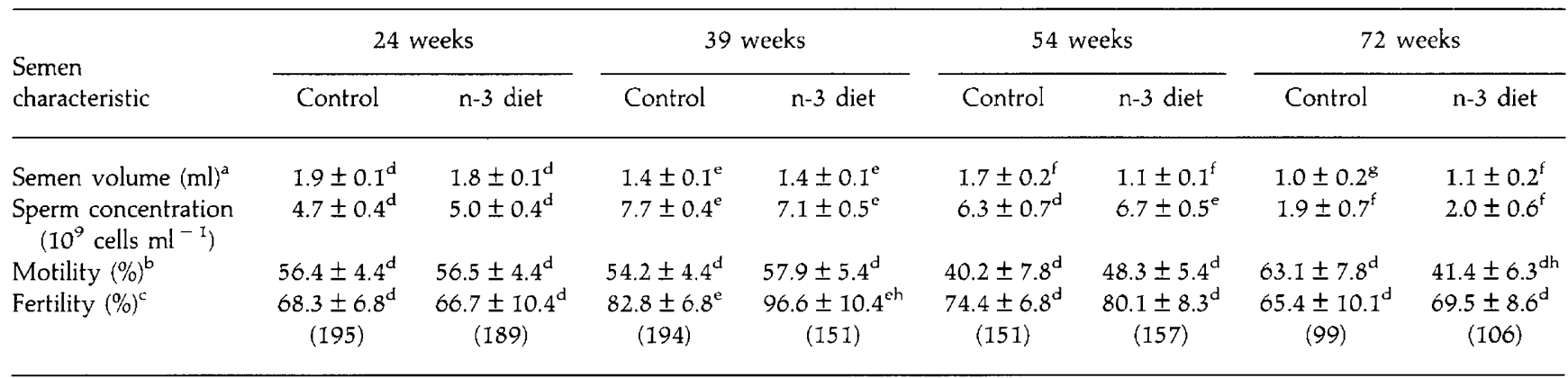

${ }^{a}$ Semen volume refers to the total volume of the pooled semen from three birds.

botility is the percentage of the total number of spermatozoa that were motile.

${ }^{c}$ Fertility is the percentage of the number of eggs (given in parentheses) containing embryos that were laid by hens artificially inseminated with the spermatozoa.

d,e.t.g For comparisons between ages for birds on the same diet, values with different superscripts are signficantly different $(P<0.05)$.

${ }^{h}$ Denotes significant difference $(P<0.05)$ between diets for birds of the same age.

Value are means $\pm \mathrm{s}$.

and the final cell pellet was resuspended in $2 \mathrm{ml} 0.85 \%(\mathrm{w} / \mathrm{v})$ $\mathrm{NaCl}$ solution.

Total lipids were extracted from the washed cell preparations after homogenization in a suitable excess of chloroform: methanoI (2:1, v:v) (Christie, 1982). The lipids were fractionated into their major classes (phospholipid, triacylglycerol, cholesterol, cholesteryl ester and free fatty acid) by thin layer chromatography on silica gel $G$ using a solvent system of hexane:diethyl ether:formic acid (80:20:1 v:v:v). After spraying the gel with 2,7-dichlorofluorescein $(0.1 \%(\mathrm{w} / \mathrm{v})$ in methanol), the bands were visualized under the UV light and the separated bands were then scraped from the plates. Phospholipid was eluted from the silica by washing it three times with $2 \mathrm{ml}$ methanol; the other lipid classes were similarly eluted using diethyl ether. The isolated lipid classes (with the exception of cholesterol) were subjected to transmethylation by refluxing for $30 \mathrm{~min}$ with methanol:toluene:sulfuric acid (20:10:1, v:v:v) in the presence of a pentadecaenoic acid (15:0) standard (Christie et al., 1970). The resultant fatty acid methyl esters were analysed by injecting $2 \mu \mathrm{l}$, via a CP9010 auto sampler (Chrompack Ltd, London), on to a Carbowax capillary column $30 \mathrm{~m} \times 0.25 \mathrm{~mm}$ wide, with a film thickness of $0.25 \mu \mathrm{m}$ (Alltech UK Ltd, Carnforth) fitted within a Chrompack CP9001 gas chromatograph (Chrompack Ltd). The fatty acid compositions (percentage $(w / w)$ of total fatty acids) were derived by integrating the peaks using an EZ Chrom data handling system (Speck Analytical Ltd, Alloa). The absolute amounts of the acyl-containing lipid classes were also calculated using this integration method (Christie et al., 1970). Cholesterol was determined by an enzymic-colorimetric method using a kit system (Boehringer Ltd, Lewes).

The individual phospholipid classes (phosphatidylcholine, phosphatidylethanolamine, phosphatidylserine, cardiolipin and sphingomyelin) were separated by high performance thin layer chromatography using a solvent system of methyl acetate:isopropanol:chloroform:methanol:0.25\% ( $w / v$ in $\mathrm{H}_{2} \mathrm{O}$ ) $\mathrm{KCl}(25: 25: 25: 19: 9, \mathrm{v}: \mathrm{v}: \mathrm{v}: \mathrm{v}: \mathrm{v})$, as described by Olsen and Henderson (1989). After charring, the phospholipids were quantified by densitometry using a Shimadzu CS-9001, dualwavelength, flying spot scanner (V. A. Howe Ltd, Banbury).

\section{Statistical analysis}

Statistical comparisons were based on 15 replicate semen samples (each sample derived from an individual bird) from each dietary group, with respect to data on semen volume, cell concentration and motility. Statistical analyses of the fertility values and of the lipid composition parameters were based on five replicate pooled semen samples from each dietary group, with each pooled sample obtained from three individual birds. The data was assessed by analysis of variance (ANOVA) using the General Linear Model method. All percentage data were arcsin transformed prior to ANOVA (Snedecor and Cochran, 1967).

\section{Results}

For the cockerels on the control diet, the concentration of spermatozoa in the semen increased significantly between 24 and 39 weeks of age and then decreased markedly between 54 and 72 weeks (Table 2). Dietary supplementation with 18:3n-3 had no effect on semen volume or sperm concentration at any stage but produced a significant decrease in motility at 72 weeks. The fertility of the semen obtained from the control birds was maximal at 39 weeks but had decreased considerably by 72 weeks. The $n-3$ supplementation resulted in a significant increase in fertility at 39 weeks (Table 2).

The concentration of total lipid in the spermatozoa cells increased with age for both the control and the birds supplemented with $18: 3 n-3$, with a significant difference between the dietary groups detected only at 72 weeks (Table 3 ). The proportion of phospholipid was maximal at 39 weeks but decreased considerably thereafter, with no differences between the dietary groups. The proportion of cholesterol was significantly higher at the later stages and at 39 and 72 weeks was increased by supplementing the diet with 18:3n-3. The concentration of free fatty acids in the spermatozoa was significantly greater at 72 weeks than at 24 weeks for the birds on the control diet. The proportion of cholesteryl ester was at a minimum at 39 weeks for both dietary groups and was also 
Table 3. The proportions of the major lipid classes in spermatozoa from cockereis on a control diet rich in linoleic acid (18:2n-6) and from birds on a diet supplemented with $\alpha$-linolenic acid $(18: 3 n-3)$ at $24,39,54$ and 72 weeks of age

\begin{tabular}{|c|c|c|c|c|c|c|c|c|}
\hline Lipid & \multicolumn{2}{|c|}{24 weeks } & \multicolumn{2}{|c|}{39 weeks } & \multicolumn{2}{|c|}{54 weeks } & \multicolumn{2}{|c|}{72 weeks } \\
\hline Phospholipid & $62.8 \pm 3.8^{\mathrm{b}}$ & $68.3 \pm 6.6^{b}$ & $69.2 \pm 2.9^{c}$ & $68.5 \pm 3.3^{b}$ & $57.3 \pm 4.6^{\mathrm{b} . \mathrm{d}}$ & $62.1 \pm 3.8^{b}$ & $51.0 \pm 3.8^{\mathrm{d}}$ & $50.8 \pm 3.8^{c}$ \\
\hline Free fatty acid & $5.9 \pm 2.1^{\mathrm{b}}$ & $7.4 \pm 3.6^{b s c}$ & $6.9 \pm 1.6^{\mathrm{b}}$ & $5.3 \pm 1.8^{c}$ & $9.1 \pm 2.6^{b, c}$ & $5.6 \pm 2.1^{c}$ & $11.4 \pm 2.1^{\mathrm{c}}$ & $10.0 \pm 2.1^{\mathrm{b}}$ \\
\hline Triacylglycerol & $7.3 \pm 1.4^{b}$ & $3.3 \pm 2.4^{b, e}$ & $3.8 \pm 1.1^{c}$ & $3.2 \pm 1.2^{b}$ & $2.5 \pm 1.7^{\mathrm{c}}$ & $2.6 \pm 1.4^{b}$ & $2.3 \pm 1.4^{\mathrm{c}}$ & $2.9 \pm 1.4^{b}$ \\
\hline
\end{tabular}

${ }^{a}$ The lipid class values refer to the percentage $(\mathrm{w} / \mathrm{w})$ of the total lipid (values are means $\pm \mathrm{so}$ ).

${ }^{b, c, d}$ For comparisons between ages for birds on the same diet, values without a superscript in common are significantly different $(P<0.05)$.

'Denotes significant difference $(P<0.05)$ between diets for birds of the same age.

Table 4. The proportions of the major phospholipid classes in spermatozoa from cockerels on a control diet rich in linoleic acid (18:2n-6) and from birds on a diet supplemented with $\alpha$-linolenic acid (18:3n-3) at 24, 39, 54 and 72 weeks of age

\begin{tabular}{|c|c|c|c|c|c|c|c|c|}
\hline \multirow[b]{2}{*}{ Phospholipid ${ }^{a}$} & \multicolumn{2}{|c|}{24 weeks } & \multicolumn{2}{|c|}{39 weeks } & \multicolumn{2}{|c|}{54 weeks } & \multicolumn{2}{|c|}{72 weeks } \\
\hline & Control & n-3 diet & Control & n-3 diet & Control & n-3 diet & Control & n-3 diet \\
\hline Phosphatidylcholine & $32.3 \pm 1.1^{\mathrm{b}}$ & $33.4 \pm 1.1^{\mathrm{b}}$ & $25.4 \pm 1.1^{\mathrm{c}}$ & $27.5 \pm 1.3^{c}$ & $34.8 \pm 1.1^{\mathrm{d}}$ & $33.2 \pm 1.3^{\mathrm{b}}$ & $42.3 \pm 1.5^{\mathrm{e}}$ & $40.0 \pm 1.3^{\mathrm{d}}$ \\
\hline Phosphatidylethanolamine & $33.1 \pm 1.4^{\mathrm{b}}$ & $31.7 \pm 1.4^{\mathrm{b}}$ & $33.8 \pm 1.4^{\mathrm{b}}$ & $32.9 \pm 1.7^{\mathrm{b}}$ & $31.1 \pm 1.4^{\mathrm{b}}$ & $32.9 \pm 1.7^{\mathrm{b}}$ & $27.4 \pm 2.0^{c}$ & $30.1 \pm 1.7^{b, f}$ \\
\hline Phosphatidylserine & $19.2 \pm 1.3^{b}$ & $18.9 \pm 1.3^{b}$ & $24.4 \pm 1.3^{c}$ & $21.8 \pm 1.5^{\mathrm{c}}$ & $20.9 \pm 1.3^{\mathrm{b}}$ & $21.1 \pm 1.5^{\mathrm{b}}$ & $11.8 \pm 1.7^{\mathrm{d}}$ & $14.1 \pm 5.4^{\mathrm{d}}$ \\
\hline
\end{tabular}

${ }^{a}$ The values refer to the percentage $(w / w)$ of the total phospholipid (values are means $\pm s v$ ).

b.c,d,c For comparisons between ages for birds on the same diet, values without a superscript in common are significantly different $(P<0.05)$.

'Denotes significant difference $(P<0.05)$ between diets for birds of the same age.

signficantly reduced at 72 weeks by the $\mathrm{n}-3$ supplementation (Table 3). The proportion of phosphatidylcholine decreased to a minimum value at 39 weeks and increased thereafter, whereas the proportion of phosphatidylserine displayed the opposite trend (Table 4). Supplementation with 18:3n-3 did not result in any significant changes in the proportions of the phospholipid classes compared with the controls, apart from an increase in phosphatidylethanolamine and a decrease in sphingomyelin at 72 weeks (Table 4 ).

The major polyunsaturated fatty acid components of the phospholipids in spermatozoa from the control birds were $20: 4 n-6$ and $22: 4 n-6$; these phospholipids were almost devoid of $\mathrm{n}-3$ polyunsaturated fatty acids apart from very low concentrations of 22:6n-3 (approximately 2\%, w/w) and $22: 5 n-3(0.5-1.0 \%, w / w)$ (Table 5). Age-related changes in these components included a significant decrease in the proportion of $22: 4 \mathrm{n}-6$ and increased concentrations of oleic (18:1n-9) and linoleic (18:2n-6) acids by 72 weeks of age. Dietary supplementation with $18: 3 n-3$ resulted in small but significant effects on these fatty acid profiles. In particular, n-3 supplementation significantly increased the proportion of $22: 5 \mathrm{n}-3$ at 39 and 54 weeks, although the maximum amount of $22: 5 \mathrm{n}-3$ thus attained was only $5.2 \%(\mathrm{w} / \mathrm{w})$ of the total phospholipid fatty acids. Most notably, no significant changes in the amount of 22:6n-3 were induced by 18:3n-3 supplementation, apart from a very small increase (from $1.9 \%$ to $2.4 \%$, $w / w)$ at week 54 and a small decrease at week 72 . However, the net effect of these small changes in the proportions of individual fatty acids as a result of 18:3n-3 supplementation was to produce considerable decreases in the $C_{20-22} n-6: n-3$ ratio at 39 and 54 weeks. (Table 6). An unforeseen consequence of supplementing the diet with n-3 was a marked increase in the concentration of saturated fatty acids, particularly stearic acid (18:0), in the sperm phospholipid at 72 weeks (Table 5). This resulted in a marked decrease in the polyunsaturated: saturated ratio at this stage (Table 6).

\section{Discussion}

The present study has revealed four main findings. First, it is apparent that age-related changes in parameters of semen quality such as sperm concentration and fertilizing ability are accompanied by a range of alterations in sperm lipid composition. Perhaps the most marked change observed in lipid composition with advancing age was the progressive increase in the amount of total lipid per cell. More specifically, the proportion of phosphatidylserine within the total phospholipid 
Table 5. The fatty acid compositions of the total phospholipid in spermatozoa from cockerels on a control diet rich in linoleic acid $(18: 2 n-6)$ and from birds on a diet supplemented with $\alpha$-linolenic acid (18:3n-3) at $24,39,54$ and 72 weeks of age

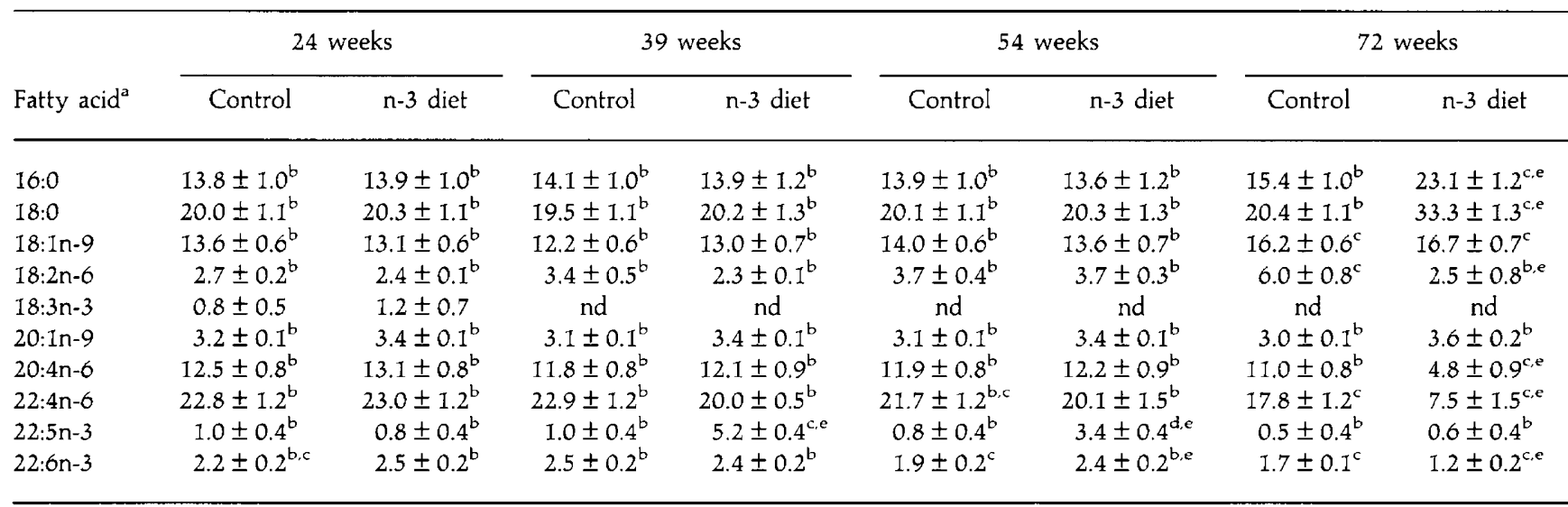

${ }^{\mathrm{a}}$ Fatty acid compositions are expressed as the percentage $(\mathrm{w} / \mathrm{w})$ of the total phospholipid fatty acids; only those fatty acids comprising $>0.5 \%(\mathrm{w} / \mathrm{w})$ are shown (values are means $\pm \mathrm{SD}$ ).

${ }_{\text {b.c.d }}$ For comparisons between ages for birds on the same diet, values without a superscript in common are significantly different $(P<0.05)$.

${ }^{\mathrm{C}}$ Denotes significant difference $(P<0.05)$ between diets for birds of the same age.

nd, not detected.

Table 6. Effects of supplementing the diet of cockerels with $\alpha$-linolenic acid (18:3n-3) on the ratios of polyunsaturated (P): saturated (S), n-6:n-3 and $C_{20-22}$ n-6:n-3 fatty acids in sperm phospholipids at $24,39,54$ and 72 weeks of age

\begin{tabular}{|c|c|c|c|c|c|c|c|c|}
\hline \multirow[b]{2}{*}{ Ratio } & \multicolumn{2}{|c|}{24 weeks } & \multicolumn{2}{|c|}{39 weeks } & \multicolumn{2}{|c|}{54 weeks } & \multicolumn{2}{|c|}{72 weeks } \\
\hline & Control & n-3 diet & Control & n-3 diet & Control & $n-3$ diet & Control & n-3 diet \\
\hline P:S & $1.2 \pm 0.1^{\mathrm{a}}$ & $1.3 \pm 0.1^{a}$ & $1.2 \pm 0.1^{\mathrm{a}}$ & $1.2 \pm 0.1^{\mathrm{a}}$ & $1.1 \pm 0.1^{\mathrm{a}}$ & $1.2 \pm 0.1^{\mathrm{a}}$ & $1.0 \pm 0.1^{\mathrm{a}}$ & $0.3 \pm 0.1^{b . d}$ \\
\hline$n-6: n-3$ & $9.5 \pm 1.3^{\mathrm{a}}$ & $8.6 \pm 1.6^{a}$ & $10.9 \pm 0.5^{a}$ & $4.5 \pm 0.6^{\mathrm{b} . \mathrm{d}}$ & $13.8 \pm 0.5^{\mathrm{b}}$ & $6.2 \pm 0.3^{\mathrm{a}, \mathrm{d}}$ & $15.8 \pm 1.4^{\mathrm{b}}$ & $8.2 \pm 3.1^{a}$ \\
\hline$C_{20-22} n-6: n-3$ & $11.0 \pm 1.5^{a . b}$ & $10.9 \pm 1.9^{a}$ & $9.9 \pm 0.4^{\mathrm{a}}$ & $4.2 \pm 0.5^{b, d}$ & $12.4 \pm 0.5^{\mathrm{b}}$ & $5.5 \pm 0.2^{\mathrm{cdd}}$ & $13.1 \pm 1.1^{b}$ & $6.8 \pm 2.7^{a, b . c}$ \\
\hline
\end{tabular}

a.b.c.For comparisons between ages for birds on the same diet, values without a superscript in common are significantly different $(P<0.05)$.

${ }^{\mathrm{d}}$ Denotes significant difference $(P<0.05)$ between diets for birds of the same age.

Values are means $\pm \mathrm{sD}$.

was maximal when fertility was high (at 39 weeks) but had decreased significantly by 72 weeks when fertility was declining. The potential importance of long-chain polyunsaturated fatty acids in sperm function was underlined by the decrease in the proportion of $22: 4 \mathrm{n}-6$ in the total phospholipid at 72 weeks of age. These changes in lipid composition with age confirm and extend previous observations (Kelso et al., 1996).

The second key result of this study is related to the question of whether the reported predominance of $n-6$ polyunsaturated fatty acids in avian sperm lipids, in contrast to the predominance of n-3 fatty acids in mammalian sperm lipids, represents a true phylogenetic difference between birds and mammals or is simply an artefact resulting from the feeding of commercial diets to domestic poultry. In general, the fatty acid compositions of the tissue lipids of animals maintained in captivity should be interpreted with some caution since the commercial diets provided may differ greatly in their fatty acid profiles from the natural diets available in the wild. In particular, such commercial diets often contain very high proportions of $n-6$ fatty acids, to the detriment of $\mathrm{n}-3$ fatty acids (Noble, 1986).
An exampie of the potential for confusion implicit in data from captive animals is provided by the laboratory guinea-pig, which, in contrast to most mammals, produces spermatozoa characterized by low proportions of 22:6n-3 and high concentrations of 22:5n-6 in the lipids of the brain and retina (Neuringer et al., 1988). Although this discrepancy was originally interpreted as a difference between species, recent work has shown that providing the guinea-pig with a diet enriched in n-3 fatty acids, as opposed to commercial chow, increases the tissue content of 22:6n-3 to typical mammalian concentrations, concomitant with the disappearance of $22: 5 n-6$ (Weisinger et al., 1995). Of greater pertinence to the present study, the low concentrations of $22: 6 n-3$ and correspondingly high concentrations of 22:5n-6 typical of the sperm lipids of the laboratory rat (in contrast to the usual pattern observed for mammalian spermatozoa) were completely reversed by replacing the laboratory chow diet with one supplemented with n-3 fatty acids. Most notably, the resultant increased amount of 22:6n-3 in the rat spermatozoa was accompanied by an improvement in testis maturation and by greater spermatogenesis (Salem et al., 1986). 
In the present work, an attempt was made to rectify any dietary deficiency of n-3 fatty acids by supplementing the feed of cockerels with linseed oil, which is rich in 18:3n-3. The aim was to determine whether such $n-3$ supplementation would result in a transition of the fatty acid profile of spermatozoa of the birds to a pattern more typical of mammals, that is, a switch from $n-6$ to n-3 fatty acids as the major components of sperm phospholipids. A major conclusion was that the fatty acid profile of the spermatozoa of the cockerels exhibits a distinct resistance to such dietary manipulation. In spite of the provision of an 18:3n-3-enriched diet to the birds for a total of some 49 weeks, little or no increase was observed in the proportion of $22: 6 n-3$, the ultimate endproduct of the chain elongation and desaturation of 18:3n-3, in the sperm phospholipids. The only stage where a significant enhancement in the concentration of 22:6n-3 was observed was at week 54 but this represented only a minor increase, from $1.9 \%$ to $2.4 \%(\mathrm{w} / \mathrm{w})$ of the total phospholipid fatty acids. This effect is trivial in comparison with the amount of this fatty acid routinely observed in mammalian spermatozoa, in which proportions of $22: 6 \mathrm{n}-3$ as high as $60-70 \%$ ( $w: w$ of total phospholipid fatty acids) are achieved in some species, even in the absence of any n-3 supplementation (Salem et al., 1986). A somewhat greater effect of the dietary supplementation with $18: 3 n-3$ was a significant increase in the proportion of $22: 5 n-3$ (the metabolic precursor of 22:6n-3) in the phospholipids at 39 and 54 weeks of age. However, the maximum concentration of $22: 5 n-3$ induced by $18: 3 n-3$ supplementation was only $5.2 \%(w: w)$ of the total phospholipid fatty acids. At all ages studied, the major polyunsaturated fatty acids in the sperm phospholipids were $20: 4 n-6$ and 22:4n-6, irrespective of the dietary supplementation. Thus, the sustained feeding of large amounts of 18:3n-3 to the cockerels completely failed to dislodge the ascendancy of $n-6$ polyunsaturates in the spermatozoa of the birds and certainly did not produce a switch to the typical mammalian pattern. The obvious conclusion from this work is that the preponderance of $n-6$ polyunsaturated fatty acids reported in the spermatozoa of domestic birds most likely reflects the natural situation and is not an aberration due to the high $n-6: n-3$ ratios typical of commercial feeds. It would appear, therefore, that the highly distinctive sperm fatty acid compositions of birds and mammals represents a clear-cut biochemical difference between these two classes of higher vertebrate.

Third, the results allow certain inferences to be drawn regarding the metabolism of polyunsaturated fatty acids in relation to spermatogenesis. There is currently a lack of information on the mechanisms involved in the delivery of fatty acyl components from the circulation to the developing spermatozoa and in the selective incorporation of specific fatty acids into the sperm phospholipids. It is clear from the present data that the desaturation and elongation of 18:3n-3 into its $\mathrm{C}_{20-22}$ derivatives, or the incorporation of these derivatives into phospholipids, is extremely inefficient in this system. Traditionally it was believed that the synthesis of $n-6$ and n-3 $C_{22}$ polyunsaturates from their $C_{18}$ precursors was performed via the sequential action of $\delta 6-, \delta 5-$ and $\delta 4$-desaturases plus the intervening elongation steps (Brenner, 1989). However, recent work using a range of mammalian cell types suggests that the direct conversion of $22: 4 n-6$ to $22: 5 n-6$ and of $22: 5 n-3$ to 22:6n-3 via the action of 84 -desaturase does not take place.
Instead, it appears that this conversion may occur via a more circuitous route involving microsomal elongation to $C_{24}$ polyunsaturates followed by retroconversion of these intermediates to $22: 5 n-6$ and $22: 6 n-3$ via peroxisomal $\beta$-oxidation (Moore et al., 1995; Sprecher et al., 1995). The present data, which indicate that dietary supplementation with $18: 3 n-3$ results in the appearance of a low concentration of $22: 5 n-3$ in the sperm phospholipid but no notable increase in the proportion of $22: 6 n-3$, suggests that the microsomal elongation of $22: 5 n-3$ and the subsequent peroxisomal retroconversion pathway does not occur in this system. This view is supported by the fact that in cockerel spermatozoa 22:4n-6 appears to be the end product of the desaturation or elongation of $18: 2 n-6$, with no further conversion to $22: 5 n-6$.

An unexpected result of supplementing the diet with $18: 3 \mathrm{n}-3$ was the increase it induced in the proportion of saturated fatty acids in the sperm phospholipid at 72 weeks of age, to the detriment of the amount of polyunsaturates of both the $n-6$ and $n-3$ series. It would appear that increasing the availability of $n-3$ fatty acids at this stage somehow inhibits the incorporation of $20: 4 n-6$ and $22: 4 n-6$ into the phospholipid without producing a corresponding increase in the incorporation of 22:5n-3 and 22:6n-3. This deficit in polyunsaturate incorporation is therefore filled by the saturated fatty acids.

The fourth finding of this study was that dietary supplementation with $18: 3 n-3$ produced a significant improvement in semen fertility (from $83 \%$ to $97 \%$ ) at week 39 . This corresponded with the increased amount of $22: 5 n-3$ and the decreased $n-6: n-3$ ratio displayed by the sperm phospholipid at this stage. Thus, although the results of this study indicate that n-6 polyunsaturates probably represent the natural major constituents of avian spermatozoa, the precise balance of $n-6: n-3$ fatty acids may be crucial to the realization of optimal fertility. It was also noteworthy that the derangement of the polyunsaturate:saturate ratio induced by the 18:3n-3-enriched diet at week 72 was associated with a significant decrease in sperm motility. In summary, the supplementation of the cockerel diet with 18:3n-3 was associated with a significant increase in the fertilizing ability of their spermatozoa at 39 weeks of age and with a corresponding increase in the proportion of n-3 fatty acids.

The authors are grateful to the Scottish Office Agriculture and Fisheries Department for financial support, to the University of Glasgow for the William Stewart Scholarship to K. A. Kelso and to Scotia Pharmaceuticals Ltd for a research fellowship to S. Cerolini.

\section{References}

Anderson GJ, Connor WE, Corliss JD and Lin DS (1989) Modulation of docosahexaenoic acid levels in the brain and retina of the newly hatched chick Journal of Lipid Research 30 433-444

Brenner RR (1989) Factors influencing fatty acid chain elongation and desaturation. In The Role of Fats in Human Nutrition pp 45-79 Eds AJ Vergroesen and $M$ Crawford. Academic Press, New York

Christie WW (1982) Isolation of lipids from tissues. In Lipid Analysis: Isolation, Separation, Identification and Structural Analysis of Lipids (2nd Edn) pp 17-25. Pergamon Press, Oxford

Christie WW, Noble RC and Moore JH (1970) Determination of lipid classes by gas chromatographic procedure Analyst 95 940-944 
Darin-Bennett A, Poulos A and White IG (1974) The phospholipids and phospholipid-bound fatty acids of dog and fowl spermatozoa journal of Reproduction and Fertility 41 471-474

Howarth B, Torregrossa D and Britton WM (1977) The phospholipid content of ejaculated fowl and turkey spermatozoa Poultry Science 56 1265-1268

Innis SM (1991) Essential fatty acids in growth and development Progress in Lipid Research 30 39-103

Jain YC and Anand SR (1976) Fatty acids and fatty aldehydes of buffalo seminal plasma and sperm lipid Journal of Reproduction and Fertility 47 261-267

Kelso KA, Cerolini S, Noble RC, Sparks NHC and Speake BK (1996) Lipid and antioxidant changes in semen of broiler fowl from 25 to 60 weeks of age Journal of Reproduction and Fertility 106 201-206

Lake PE and Stewart JM (1978) AI in poultry Bulletin 213 Ministry of Agriculture, Fisheries and Food, London

Lin DS, Connor WE, Wolf DP, Neuringer M and Hachey DL (1993) Unique lipids of primate spermatozoa: desmosterol and DHA Joumal of Lipid Research 34 491-499

Moore SA, Hurt E, Yoder E, Sprecher H and Spector A (1995) Docosahexaenoic acid synthesis in human skin fibroblasts involves peroxisomal retroconversion of tetrahexaenoic acid Journal of Lipid Research 36 2433-2443

Neill AR and Masters CJ (1972) Metabolism of fatty acids by bovine spermatozoa Biochemical Journal 127 375-385

Neuringer M, Anderson GJ and Connor WE (1988) The essentiality of n-3 fatty acids for the development and function of the retina and brain Annual Review of Nutrition 8 517-541

Nissen HP and Kreysel HW (1983) Polyunsaturated fatty acids in relation to sperm motility Andrologia 15 264-269

Nissen HP, Kreysel HW and Schirren C (1981) Composition of the lipid-bound fatty acids of human semen in relation to its fertility values Andrologia 13 $444-451$
Noble RC (1986) Egg lipids. In Egg Quality: Current Patterns and Recent Advances pp 159-177 Eds RG Wills and CG Belyavin. Butterworths, London

Olsen RE and Henderson RJ (1989) The rapid analysis of neutral and polar marine lipids using double-developed HPTLC and scanning densitometry Journal of Experimental Marine Biology and Ecology 129 189-197

Poulos A and White IG (1973) The phospholipid composition of human spermatozoa and seminal plasma Journal of Reproduction and Fertility 35 265-272

Poulos A, Darin-Bennett A and White IG (1973) The phospholipid-bound fatty acids and aldehydes of mammalian spermatozoa Comparative Biochemistry and Physiology 46B 541-549

Ravie $O$ and Lake PE (1985) The phospholipid-bound fatty acids of fowl and turkey spermatozoa Animal Reproduction Science 9 189-192

Salem N, Kim HY and Yergey JA (1986) Docosahexaenoic acid: membrane function and metabolism. In Health Effects of Polyunsaturated Fatty Acids in Seafoods pp 263-321 Eds AP Simopoulos, RR Kifer and RE Martin. Academic Press, New York

Sanders TAB (1988) Essential and trans-fatty acids in nutrition Nutrition Research Reviews 1 57-78

Scott TW (1973) Lipid metabolism of spermatozoa Joumal of Reproduction and Fertility 18 65-76

Snedecor GW and Cochran WG (1967) Two way classifications. In Statistical Methods (6th Edn) pp 299-334. lowa State University Press, Ames

Sprecher H, Luthria Dr Mohammed BS and Baykousheva SP (1995) Re-evaluation of the pathways of the biosynthesis of polyunsaturated fatty acids Journal of Lipid Research 36 2471-2477

Weisinger HS, Vingys AJ and Sinclair AJ (1995) Dietary manipulation of long chain polyunsaturated fatty acids in the retina and brain of guinea pigs Lipids $30471-473$ 